\title{
NUMERICAL DETERMINATION OF THE VARIATION WIDTH OF FEASIBLE ODFS
}

\author{
H. SCHAEBEN \\ Laboratory of Metallurgy of Polycrystalline Materials (LM2P), University of \\ Metz, Ile du Saulcy, 57045 Metz, France
}

(17 June 1992)

Within all known methods of calculating a model orientation density (odf) from a given set of pole densities (pdfs) the set of all feasible odfs and in particular its associated variation width remained unaccessible up to now.

Here, the variation width is mathematically well defined for the continuous and the discrete setting of the fundamental projection equation of texture goniometry. For the discrete case it is shown that it can be numerically determined by a sequence of optimization problems with extremely simple objective functions.

The general solution is exemplified for the $S A N T A F E$ model data.

KEY WORDS Ill-posed inversion problem of texture goniometry, feasible odfs, variation width, mathematical programming, linear programming.

\section{THE ILL-POSED INVERSION PROBLEM OF TEXTURE GONIOMETRY}

The diffraction experiment of texture goniometry is mathematically described in terms of continuous probability density functions by the Fredholm-type 1 integral equation

$$
\tilde{P}_{\mathbf{h}}(\mathbf{r})=C \int_{G} K(\{\mathbf{h}\}, \mathbf{r} ; g) f(g) d v(g)
$$

with $\mathbf{r}, \mathbf{h} \in S_{+}^{3}, g \in G$, and $d v(g)$ an infinitesimal volume element of $G$ containing $g$, and some normalization factor $C>0$; or in terms of discrete probabilities by the corresponding system of linear equations

$$
\boldsymbol{\pi}(\mathbf{h}) \mathbf{x}=\mathbf{y}(\mathbf{h})
$$

with $x_{n} \geq 0$ for all $n=1, \ldots, N, \sum_{n=1}^{N} x_{n}=1$, and with

$$
\begin{gathered}
\pi_{p n}(\mathbf{h})=C \int_{Z_{p}} \int_{G_{n}} \sum_{m=1}^{M_{k} / 2} 1_{G_{\mathrm{h}_{m}}(\mathbf{r})}(g) d v(g) d s(\mathbf{r}) \\
x_{n}=\int_{G_{n}} f(g) d v(g) \geq 0, \quad n=1, \ldots, N \\
y_{p}(\mathbf{h})=\int_{Z_{p}} \tilde{P}_{\mathbf{h}}(\mathbf{r}) d s(\mathbf{r}) \geq 0, \quad p=1, \ldots, P
\end{gathered}
$$

cf. Schaeben $(1988,1991)$. 
Initially, the mathematical problem of quantitative texture analysis (QTA) was thought to calculate the orientation density (odf) $\hat{f}$ from a given set $\mathscr{P}$ of experimental pole density functions $\tilde{P}_{\mathrm{h}}$ of crystal forms $\{\mathbf{h}\}$, i.e. to find the unique solution of Eq. (1), respectively of Eq. (2).

Since it was understood that this tomographic inversion problem does not possess a unique solution but infinitly many different solutions (Matthies, 1979), much effort has been spent to develop methods, algorithms, and computer codes, based on heuristics and/or on well defined mathematical models to calculate an odf from given pdfs which

- explains the available pdfs, and

- is non-negative and satisfies the constraints given by Eq. (1), respectively Eq. (2), (4);

- is physically reasonable, i.e. provides a conservative interpretation;

- is mathematically sensible and numerically accessible at reasonable computational costs.

Summarily, all known pdf-to-odf inversion methods approximate a particular odf $\hat{f}$ of the infinite set $\mathscr{F}$ of all feasible odfs. This particular model solution is specified by some additional mathematical assumption, usually by some optimality criterion, e.g. least squares fit of harmonics, minimum or maximum texture index $\left(l^{2}\right.$-norm), maximum entropy etc., or just by the algorithm used to actually calculate it, e.g. harmonic positivity, $h^{2}$ - or exp-approach, generalized Bayesian formula, or vector method.

\section{VARIATION WIDTH OF FEASIBLE ODFS}

Within these various methods the variation width of all feasible odfs remained essentially unaccessible. Adapting the continuous notation of Eq. (1), the variation width is defined pointwise for each $g \in G$ by

with

$$
0 \leq l(g) \leq f(g) \leq u(g), \quad g \in G
$$

$$
\begin{aligned}
& l(g)=\inf _{f \in \mathscr{F}} f(g), g \in G \\
& u(g)=\sup _{f \in \mathscr{F}} f(g), g \in G
\end{aligned}
$$

where $\mathscr{F}$ denotes the set of all feasible odfs with respect to a given set $\mathscr{P}$ of experimental pdfs $\tilde{P}_{\mathbf{h}}$ of crystal forms $\{\mathbf{h}\}$. It should be noted that the functions $l(g)$ and $u(g)$ are not probability densities, and hence not orientation densities. Furthermore, it is emphasized that they depend on the set $\mathscr{P}$ of pdfs.

In the discrete setting of Eq. (2) the variation width is defined by

$$
0 \leq l_{n} \leq x_{n} \leq u_{n}, \quad n=1, \ldots, N
$$


where $\mathbf{I}$ and $\mathbf{u}$ are given by the sequence of optimization problems $\min F_{n}(x)=\min \mathbf{e}_{n} \mathbf{x}=l_{n}$, resp. $\max F_{n}(x)=\max \mathbf{e}_{n} \mathbf{x}=u_{n}, \quad n=1, \ldots, N$

$$
\text { subject to } \pi(\mathbf{h}) \mathbf{x}=\mathbf{y}(\mathbf{h}), \quad x_{n} \geq 0, \quad n=1, \ldots, N, \quad \sum_{n=1}^{N} x_{n}=1
$$

where $e_{n}$ denotes the $n$-th unit vector of $I R^{N}$.

Now, the bounds $I$ and $u$ depend on the given set $\mathscr{P}$ of pdfs and the discretization, i.e. on the partitions $\left(Z_{p}\right), p=1, \ldots, P$, of the hemisphere $S_{+}^{3} \subset I R^{3}$ of poles, and $\left(G_{n}\right), n=1, \ldots, N$, of the (Eulerian) orientation space $G \subset S O(3)$.

Each individual optimization problem of the sequence (10) is a problem of linear programming (LP) with an extremely simple linear objective function, and can be solved numerically with approriate software (Murtagh and Saunders, 1978); however, it should be remembered that the matrix $\pi$ is of large rank deficiency (Schaeben, 1984).

In terms of elementary geometry the $2 \times N$ vertices of some simplex in $[0,1]^{N}$ with faces given by the linear equations of system (2) are to be determined.

\section{AN ELEMENTARY EXAMPLE}

To gain some more insight in the geometrical aspects a little example $A \mathbf{z}=\mathbf{b}$ is studied and solved graphically. Taking

$$
A=\left(\begin{array}{lllc}
0.89 & -0.53 & 0 & 0 \\
0.47 & -0.28 & 0.89 & -0.53 \\
0 & 0 & 0.47 & -0.28
\end{array}\right)
$$

dropping the normalization because here it is neither essential nor helpful, and setting

$$
\mathbf{b}=\left(\begin{array}{l}
0.36 \\
0.55 \\
0.19
\end{array}\right)
$$

one immediately reads off the particular solution $z_{1}=z_{2}=z_{3}=z_{4}=1$. However, due to the rank deficiency of the matrix $A$ there are infinitly many solutions; the set of all solutions may be described in the form

$$
\begin{aligned}
& z_{2}=\frac{89}{53} z_{1}-\frac{36}{53} \\
& z_{3}=\frac{28}{53} z_{1}+\frac{25}{53} \\
& z_{4}=\frac{47}{53} z_{1}+\frac{6}{53}
\end{aligned}
$$




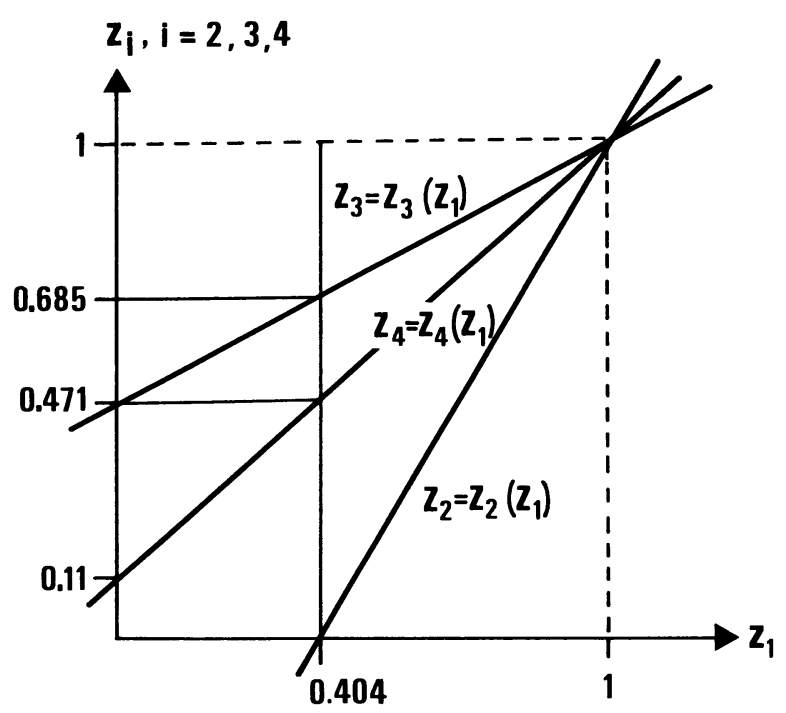

Figure 1 Graphical determination of the variation width of the set of solutions of the system of linear equations given by Eqs. (12), (13).

with $z_{1}$ arbitrarily varying in $\mathbb{R}$. Considering now the additional non-negativity constraints $0 \leq z_{n} \leq 1, n=1, \ldots, 4$, implies

$$
\begin{aligned}
0.404 & \leq z_{1} \leq 1 \\
0 & \leq z_{2} \leq 1 \\
0.685 & \leq z_{3} \leq 1 \\
0.471 & \leq z_{4} \leq 1
\end{aligned}
$$

The graphical solution is depicted in Figure 1.

\section{PRACTICAL APPLICATION TO THE SANTA FE MODEL PROBLEM}

The sequence of optimization problems (10) has been solved for the set of mathematical model-pds and -odf, respectively, devised by Matthies (1988) and referred to as SANTA FE problem (Figure 2). For the actual calculations, the three incomplete pdfs of the crystal forms (100), (110), and (111) arbitrarily truncated at 75 degrees polar angle were used as right hand sides of Eq. (2), and the same partitions of $S_{+}^{3}$ and $G$, respectively, which were used to calculate the feasible odf with maximum entropy with the program package MENTEX (Schaeben, 1991), yielding values of $R P 0$ and $R P 1$ of magnitude 0.5 .

The lower bounds $l_{n}$ of $x_{n}$ (cf. Eq. (9)) are $l_{n}=0$ for all $n=1, \ldots, N$. It is suggested that this feature is an implication of the large column rank deficiency of the matrices $\pi(\mathbf{h})$ and may be proved theoretically by means of linear algebra. The upper bounds $u_{n}$ of $x_{n}$ are displayed in Figure 3 analogously to an odf. Their 
smallest, mean, and largest values are $1.46,10.23,18.18$. The smallest, mean, and largest differences between the MENTEX model solution $x_{n}$ and the lower bounds $l_{n}$, and the upper bounds $u_{n}$ respectively, are $0.67,1.0,5.26$, and 0.63 , $9.23,17.27$ respectively.

As was emphasized above, the bounds $I$ and $\mathbf{u}$ depend on the given set $\mathscr{P}$ of pdfs (100), (110), and (111), and on the partitions $\left(Z_{p}\right), p=1, \ldots, P$, and $\left(G_{n}\right)$, $n=1, \ldots, N$. Using pdfs of crystal forms with large crystal multiplicity, e.g. (311), would impose more restrictive constraints on the upper bounds $u_{n}$ and would possibly result in smaller numerical values of $u_{n}$. However, in this first numerical study of the variation width of feasible odfs those pdfs were used which are preferrably used for pdf-to-odf inversion.

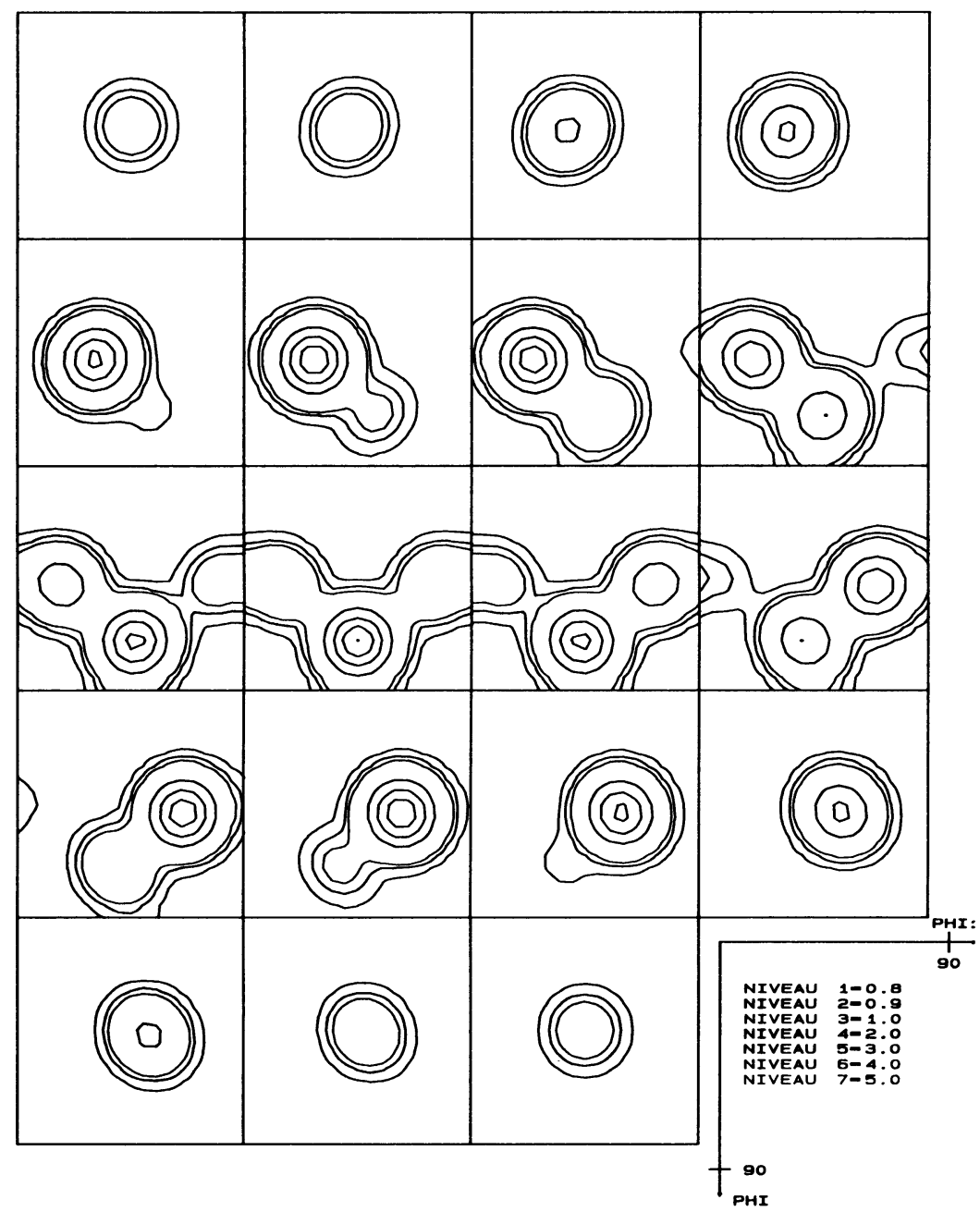

Figure 2 (a) Mathematical model odf $f_{\text {SANTAFE }}$. 


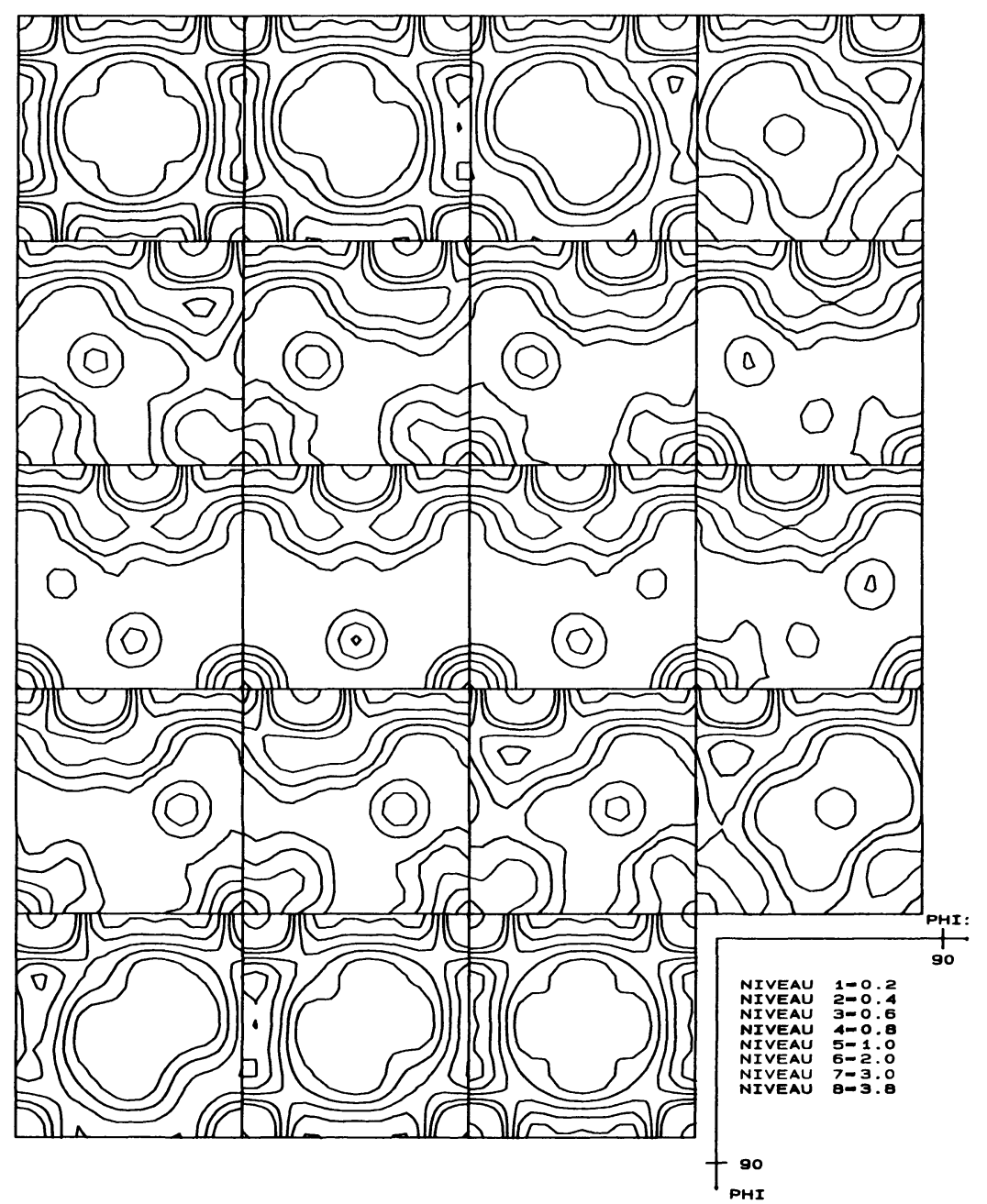

Figure 2 (b) Corresponding even mathematical model function $\tilde{f}_{\text {SANTAFE }}$.

It might also be tempting to systematically study the influence of a prior estimate $\hat{\alpha}$ of the uniform portion, or minimum value of the odf respectively, particularly on the upper bounds $u_{n}$. This would require to solve the sequence (10) of optimization problems subject to

$$
\pi(\mathbf{h}) \mathbf{x}=\mathbf{y}(\mathbf{h}), \quad x_{n} \geq \hat{\alpha}, \quad n=1, \ldots, N, \quad \sum_{n=1}^{N} x_{n}=1
$$

for various values of $\hat{\alpha}$. However, cpu-time may prove prohibitive. 


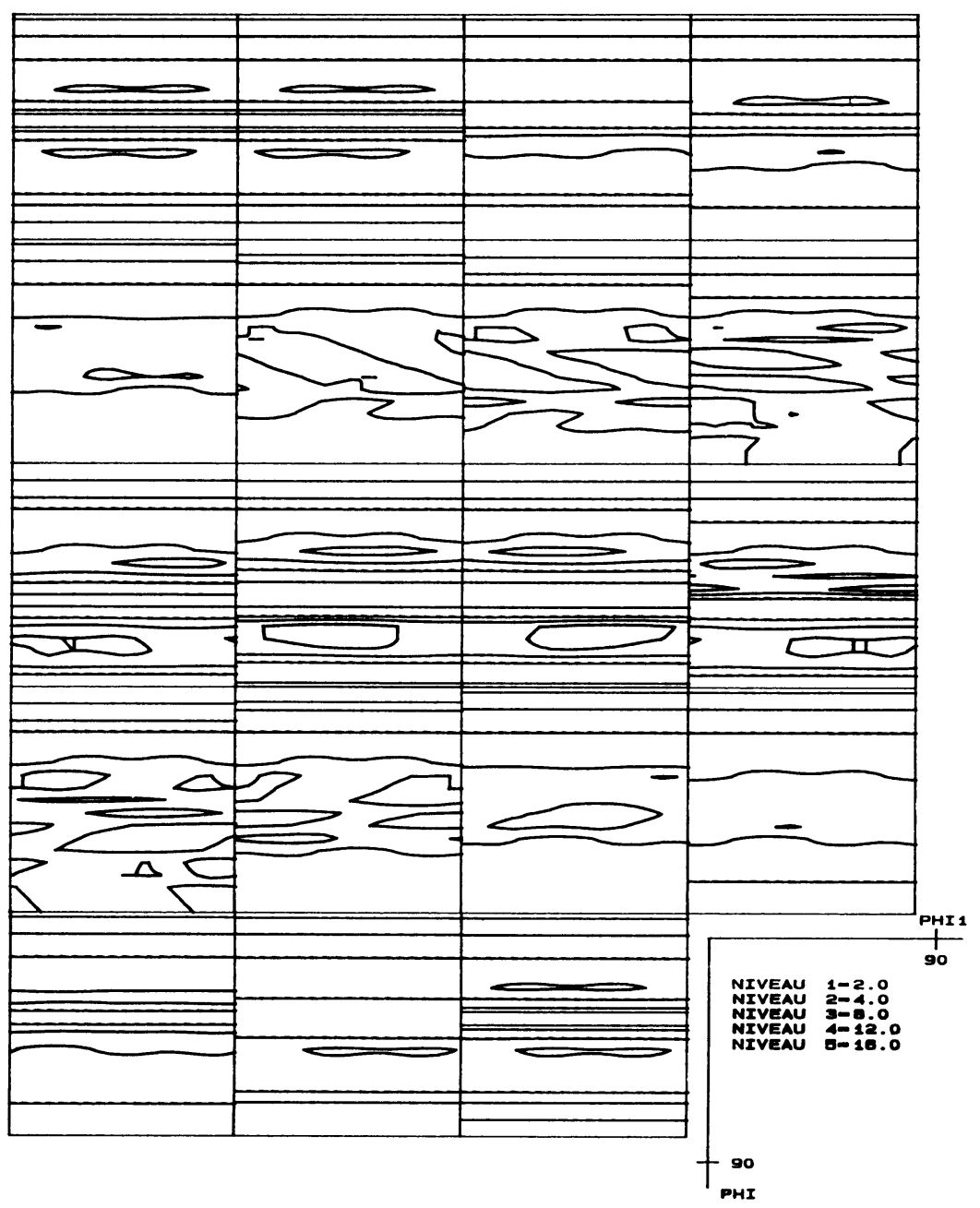

Figure 3 Upper bounds $b_{n}$ in $\varphi_{2}$-sections of conventional odf display in Eulerian orientation space.

\section{CONCLUSIONS}

For the present, these first results may be summarized that the additionally required heuristic or mathematical modeling assumptions to specify a particular feasible odf are essential for its conservative interpretation and its application in truly quantitative calculations of anisotropic properties. They also confirm that the $M E N T E X$ model solution provides a conservative approximation of the given orientation density of the SANTA FE problem.

In many practical applications it may be sufficient to solve some individual max-problems of the sequence (10) of LP problems for specific values $n_{0}$, $1 \leq n_{0} \leq N$, e.g. to gain some insight in how much confidence the global maximum deserves. 


\section{ACKNOWLEDGMENTS}

This research was funded by a grant for proposition no. ERB4040PL900089 from the EC plan SCIENCE under contract no ERBSC1*CT005001. Special thank is due to Michael A. Saunders, Systems Optimization Laboratory, Stanford, CA, for providing access to the appropriate software and his support on all numerical aspects involved in the communicated results, and Jean-Jaques Fundenberger, LM2P, Metz, for his invaluable assistance in all matters of computing and computing machinery.

\section{References}

[1] Matthies, S. (1979). Phys. Stat. Sol. (b), 92, K135-K138.

[2] Matthies, S. (1988). In Kallend, J. S. and Gottsein, G. (eds.) Proceedings 8th International Conference of Textures of Materials (ICOTOM8), Sante Fe, NM, USA, Sept. 87, The Metallurgical Society, 37-48.

[3] Murtagh, B. A. and Saunders, M. A. (1978). Mathematical Programming, 14, 41-72.

[4] Schaeben, H. (1984). Phys. Stat. Sol. (b), 123, 425-434.

[5] Schaeben, H. (1988). Phys. Stat. sol. (b), 148, 63-72.

[6] Schaeben, H. (1991). J. Appl. Phys., 69, 1320-1329. 